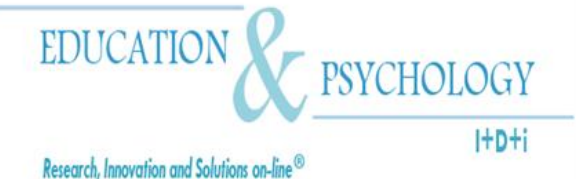

\title{
Análisis de las relaciones entre creatividad y altas capacidades en Primaria y Secundaria
}

\section{Montserrat Marugán de Miguelsanz ${ }^{1}$ Miguel Ángel Carbonero Martín ${ }^{1}$ $M^{\text {a }}$ del Henar Torres González ${ }^{1}$ Benito León del Barco ${ }^{2}$}

\author{
${ }^{1}$ Departamento de Psicología. Universidad de Valladolid \\ ${ }^{2}$ Departamento de Psicología y Antropología. Universidad de Extremadura
}

\section{España}

Correspondencia: Montserrat Marugán de Miguelsanz. Facultad de Educación y Trabajo Social. Paseo de Belén, $n^{\circ}$ 1.47011. Valladolid. España. E-mail: mmarugan@psi.uva

(C) Education \& Psychology I+D+i and Editorial EOS (Spain) 


\section{Resumen}

Introducción. En muchos modelos teóricos la creatividad es un elemento básico para definir la superdotación. Se ha efectuado una amplia revisión de investigaciones precedentes sobre el tema. Además, sobre una muestra de alumnos de Primaria y Secundaria se examinan las diferencias en las habilidades creativas, globales y por factores, entre el grupo de alumnos altamente capacitados y el resto de la muestra. Se ha intentado determinar si todos los sujetos de alta capacidad intelectual obtienen resultados elevados en una prueba de creatividad gráfica.

Método. La muestra estuvo compuesta por 477 estudiantes de $4^{\circ}, 5^{\circ}$ y $6^{\circ}$ de Primaria y de $1^{\circ}$ y $2^{\circ}$ de Secundaria de dos centros públicos y dos concertados. Se detectaron 54 sujetos de alta capacidad intelectual (CI igual o superior al percentil 95 en una prueba de inteligencia general no verbal). Se analizan los resultados en nueve factores de creatividad a través de la ejecución en una prueba de creatividad gráfica y cuantificable.

Resultados. Los sujetos de alta capacidad intelectual obtuvieron unas puntuaciones medias superiores en la prueba de creatividad y en todos los factores de la misma; sin embargo sólo un subgrupo de los sujetos bien dotados obtiene un percentil superior a 75. Así mismo se ha encontrado un amplio grupo de alumnos que sin ser de altas capacidades, supera en creatividad de forma significativa al resto de la muestra de alta dotación.

Discusión y conclusiones. Estos resultados siguen la línea de los estudios clásicos sobre creatividad en los que las correlaciones entre esta variable y la inteligencia general, eran entre débiles y moderadas, según el campo creativo estudiado. Nuestro estudio sugiere que una buena capacidad intelectual permite mejores resultados en la resolución de tareas creativas, pero no todos los sujetos de alta capacidad intelectual son excepcionalmente creativos, pudiendo tener un alto nivel en esta variable alumnos con puntuaciones menores en CI.

Palabras clave: creatividad; alta capacidad intelectual; superdotación; test de creatividad; evaluación psicométrica. 


\title{
Analysis of connections between creativity and high capacities
}

\begin{abstract}
Introduction. Due to in many theoretical models creativity is a basic element in order to define intellectual giftedness, in this work, relations between creative abilities and intellectual capacity are examined. At this end, as a first step, we have developed a wide review of previous investigations on the subject. On the basis of a sample containing students belonging to Primary and Secondary Schools, global or by factors differences in creative abilities are analized between gifted students and the remainder ones. Finally, we have tried to determine if the former get high marks when doing a graphical test.
\end{abstract}

Method. The study has been developed on the basis of a sample of 447 students belonging to $4^{\circ}, 5^{\circ}$ and $6^{\circ}$ levels of Primary School and to $4^{\circ}$ and $5^{\circ}$ levels of Secondary Education. Four schools, both public and delivered via a public private partnership, were considered. 54 students were found to have an intelligence quotient (IQ) equal or higher than the 95 percentile in a non-verbal intelligence test. We investigate numerical results obtained by the students on 9 creativity factors, for a proposed graphical test of creativity.

Results. Students with high intellectual capacity had better average results, statistically meaningful, in the creativity test and in all its factors. Nevertheless, only a part of these students had very good scores (percentile higher than 75). On the other hand, a large number of students without high intellectual abilities exceeds in creativity to the remainder gifted students. These results are also statistically meaningful.

Discussion and conclusions. Results are in agreement with those obtained in classical investigations where, depending on the creativity field studied, weak or moderate correlations between IQ and creativity were found. The present work suggests that a high intellectual capacity leads to better results when solving creativity tasks. However, students with lower IQ can show a high creativity, as not all gifted students are exceptionally creative

Keywords: creativity; high intellectual ability; intellectual giftedness; creativity test; psychometric evaluation.

Received: 02/02/12 Initial acceptance: 04/17/12 Final acceptance: $11 / 17 / 12$ 


\section{Introducción}

El interés de la psicología por la creatividad se inició en los años 50, hoy en día sigue siendo un tema de actualidad ligado a las nuevas exigencias del mercado laboral; además el desarrollo de las capacidades creativas y la atención integral a todos los alumnos, incluidos los más dotados, se han convertido en objetivos formales de los sistemas educativos. La creatividad es una facultad humana habitualmente asociada a la inteligencia, importantes modelos teóricos sobre superdotación incluyen a esta variable como uno de los rasgos que definen a la persona superdotada. Según Sternberg (1990) una de las características de la superdotación es la capacidad para encontrar nuevas soluciones a los problemas (insight). Renzulli (1999), en su ya clásico modelo de la puerta giratoria o de los tres anillos, considera la creatividad uno de los rasgos definitorios de la superdotación, junto con la alta capacidad intelectual y la perseverancia en la tarea.

Las diversas teorías admiten que inteligencia y creatividad son dos facultades diferentes pero relacionadas, y en función de esta interrelación se establecen tres posturas distintas: a) creatividad e inteligencia son interdependientes, de modo que ambas estarían al mismo nivel; b) la creatividad se considera un elemento de la inteligencia, ya que ésta sería la capacidad básica de la que la creatividad es resultado y c) la inteligencia es un componente de la creatividad, en el sentido de que la creatividad precisa de la inteligencia (Alonso, 2000). En todo caso, los planteamientos actuales sobre la creatividad son multidimensionales. Se considera que surge de la interacción de múltiples factores, tanto individuales (cognitivos, emocionales, motivacionales) como contextuales (ambiente, grupo social). Sternberg y Lubart (1997) consideran que es una capacidad que todo el mundo posee en cierta medida. Según su propuesta, en un producto creativo han de confluir seis factores: la capacidad cognitiva, el conocimiento, los estilos de pensamiento, la personalidad, la motivación y el ambiente.

Las investigaciones cuyo objetivo ha sido relacionar inteligencia (habitualmente medida en términos de CI) y creatividad son numerosas. Guilford y Hoepfner (1967) encontraron correlaciones moderadas entre pruebas de pensamiento divergente y el CI en estudiantes. Sus datos adoptaban una forma triangular en un diagrama de dispersión. Esa forma triangular indicaba que los estudiantes de CI bajo puntuaban también bajo en las pruebas de pensamiento divergente. Sin embargo, las puntuaciones de los estudiantes de CI alto se dispersaban, con puntuaciones bajas y altas en este tipo de pensamiento. 
Son muy conocidos los estudios realizados durante los años 50 y 60 del pasado siglo por el Institute of Personality Assessment and Research (IPAR), de la Universidad de California. Muchos de sus hallazgos cuentan todavía con un gran respaldo. Sternberg y O’hara (1999), basándose en Barron y Harrington (1981), resumen las principales conclusiones de este Instituto sobre la relación entre inteligencia, medida en CI, y creatividad. En primer lugar, las personas creativas suelen tener un CI por encima de la media, aunque este CI no debe interpretarse como un punto de corte, es más la expresión del hecho de que las personas con un CI bajo o medio no suelen alcanzar los niveles de los individuos muy creativos. En segundo lugar, un CI superior a 120 no parece tener tanta influencia en la creatividad como un CI inferior. La creatividad correlaciona en mayor grado con el CI inferior a 120 y en menor grado si éste es superior. A partir de un CI de 120 las correlaciones son desde débiles a inexistentes. Sternberg y Lubart (1996) sugieren incluso que un CI excesivamente alto puede interferir en la creatividad, ya que en estas personas las habilidades analíticas (las típicamente medidas por los tests de inteligencia) están tan desarrolladas que pueden frenar su potencial creativo. Esta relación entre CI y creatividad suele conocerse como "Teoría del umbral": para ser creativo se necesita un cierto nivel de CI, sin que esto implique que un CI alto equivalga a alta creatividad. En otras palabras, las personas altamente creativas generalmente poseen un CI alto, pero no todas las personas con CI alto son extremadamente creativas. En último lugar, se admite que la correlación entre CI y creatividad es variable. Generalmente oscila entre débil y moderada. Esta variación depende, por una parte, de los aspectos de inteligencia y creatividad que se midan y de los instrumentos empleados para ello, y, por otra parte, del dominio en que trabajan las personas estudiadas, puesto que el papel de la inteligencia no es el mismo en las matemáticas o en la ciencia que en las artes o la música. Por ejemplo, Barron (1981), citado por Sternberg y O’hara (1999, pp. 261) encontró una correlación cercana a cero (- .08) en un estudio con arquitectos y otra ligeramente superior en un estudio con militares (.33). Torrance (1975) revisó 388 correlaciones entre inteligencia y creatividad de una gran variedad de publicaciones. Su conclusión fue que las dos variables correlacionan sólo moderadamente. Si la evaluación de la creatividad incluía sólo pruebas figurativas la correlación media era de .06; si incluía sólo tareas verbales era de .20 y si se había empleado ambos tipos de prueba, la correlación media entre CI y creatividad era de .21.

Aunque las investigaciones no son concluyentes, se admite una interrelación mayor entre inteligencia y creatividad, cuando se refiere a los ámbitos verbales y más débil respecto 
a los figurativos. Matud (1999), con 118 estudiantes de Educación Superior en los que valoró inteligencia general y creatividad verbal y gráfica, encontró una correlación significativa, de intensidad leve, entre la puntuación de creatividad gráfica y la inteligencia. En su trabajo, el índice global de creatividad y el de creatividad verbal no correlacionaron con el CI de forma significativa. Sternberg y Lubart (1997) aplicaron una prueba de creación en cuatro dominios: escritura, perspectiva espacial, publicidad y ciencia. Encontraron correlaciones moderadas entre la puntuación de creatividad y los resultados de un test de inteligencia fluida en todos los dominios. Ferrando, Prieto, Ferrandiz y Sánchez (2005), apuntan bajas correlaciones entre inteligencia y creatividad, aunque éstas aumentan si la relación se establece con las inteligencias múltiples. Según los autores la Teoría del umbral no se constata cuando se relaciona el CI con la creatividad, aunque sí lo hace con la inteligencia contemplada desde una perspectiva multidimensional, concretamente para la inteligencia viso-espacial, corporal, naturista y lingüística.

Algunas investigaciones actuales han cuestionado las hipótesis clásicas sobre la relación entre ambas facultades. Preckel, Holling y Wiese (2006) realizaron un estudio con más de 1300 adolescentes entre 12 y 16 años para comprobar empíricamente la Teoría del umbral. Aplicaron una prueba de inteligencia fluida (Test de factor "g") y tareas de creatividad en tres dominios: verbal, figurativo y numérico. Las correlaciones para toda la muestra fueron moderadas con la creatividad global y verbal, mientras que fueron bajas en creatividad figurativa y numérica. Al analizar los resultados en los grupos según si su CI era superior o inferior a 120, hallaron que las correlaciones eran prácticamente idénticas en ambos grupos. Por ejemplo, la correlación entre creatividad global y CI fue de 0,42 en el grupo de CI menor a 120 y de 0,38 en el grupo de CI superior. Su conclusión es que la Teoría del umbral no es apoyada por los datos, por lo menos en las edades del estudio. La asociación entre la creatividad, global y de dominio, y el CI era similar en ambos grupos de capacidad. Conclusiones similares han sido alcanzadas en otras experiencias. Sligh, Conners y Roskos (2005) trabajaron con estudiantes de los primeros años de universidad. Según sus resultados, de nuevo, la correlación entre CI y creatividad era igual de alta en ambos grupos de CI. Comprobaron la relación por separado con inteligencia fluida y cristalizada. Los datos eran contrarios a la Teoría del umbral en inteligencia cristalizada y mostraron un umbral inverso en inteligencia fluida (menos relación entre CI y creatividad a partir de un CI inferior a 120). Se han encontrado, también, correlaciones entre creatividad e inteligencia emocional (Ademola, Akintunde y Yakasi, 2010). 
La vinculación de la creatividad con la superdotación se ha realizado contrastando la ejecución en tareas creativas de superdotados, o sujetos con altas capacidades, con grupos de inteligencia media. Los ámbitos en los que se ha comparado la capacidad creativa de ambos grupos han sido muy variados. Bermejo, Castejón y Sternberg (1996) emplearon siete tipos de tareas de razonamiento lógico que requieren altas dosis de insight o resolución creativa (Davidson y Sternberg, 1986). En la Teoría triárquica de la inteligencia de Sternberg (1985), el insight es el proceso intelectual que diferencia en mayor grado a los sujetos superdotados de los que no lo son. Las tareas eran: problemas matemáticos, problemas verbales, misterios, series de letras, analogías verbales, encontrar pistas y problemas de codificación selectiva. Aplicaron estas tareas y dos pruebas de inteligencia (Test de factor "g" de Cattell y el STAT, Sternberg's Triarchic Ability Test) en una muestra de 1125 niños de 8 y 9 años. El grupo de sujetos de alta capacidad intelectual, con resultados superiores al percentil 90 en una o ambas pruebas de inteligencia, obtuvo unos resultados medios superiores, con una diferencia significativa, en todas las tareas excepto la de resolución de misterios.

Ward, Saunders y Dodds (1999), desde una perspectiva interesada por la creatividad como proceso cognitivo, propusieron una tarea de creación imaginativa a un grupo de adolescentes superdotados. La actividad consistía en imaginar y dibujar una fruta que pudiera existir en otro planeta. La muestra estaba formada por 54 superdotados y un grupo control de 100 estudiantes. El grado de elaboración (características y detalles imaginados) era similar en ambos grupos. El grupo control realizó creaciones menos fantásticas, más cercanas a la realidad, y menos originales que el grupo de superdotados. López (2001) llevó a cabo un estudio descriptivo de la creatividad en un grupo de 100 alumnos precoces de Educación Infantil (5 años) y Educación Primaria (6 años). Los instrumentos empleados para valorar creatividad fueron el Test de Pensamiento Creativo de Torrance (TTCT) y el cuestionario de Martínez y Rimm (1985). Comparó los resultados obtenidos por el grupo de alto nivel intelectual y un grupo contraste de nivel medio. En Educación Infantil no encontraron diferencias significativas entre ambos grupos en ninguno de los resultados del TTCT (fluidez, flexibilidad, originalidad, elaboración y total). En cambio, en Educación Primaria se detectaron diferencias significativas, a favor del grupo de alta capacidad, en todos los resultados del TTCT excepto en elaboración. Además, encontraron que la característica "Interés" del cuestionario de Martínez y Rimm arrojaba diferencias significativas en Infantil y Primaria, mientras que en las otras características valoradas (independencia e imaginación) no existían diferencias entre ambos 
grupos. Los resultados de las investigaciones realizadas sugieren que los sujetos más capaces intelectualmente se muestran más creativos en diversos ámbitos, o al menos, lo son en algunos factores de creatividad como la originalidad, la fantasía o la fluidez (generación de múltiples respuestas).

De los datos hasta ahora revisados se desprende que es poco lo que puede afirmarse con rotundidad de la relación entre ambas capacidades. Sí es cierto que goza de bastante consenso la perspectiva de cierta interrelación entre ambas, así como la Teoría del umbral (Monreal, 2000, pp. 164 - 166), sin embargo, los datos de la investigación son, cuanto menos, dispares. Este, no obstante, podría ser el lado positivo: es una interesante cuestión de investigación aún abierta, para la que se necesitan respuestas (Sternberg y O’’hara, 1999, pp. 269).

El estudio que se expone forma parte de un proyecto más amplio: "Intervención educativa con niños superdotados en Castilla y León", en el marco del Grupo de Investigación de Excelencia GR179 de Psicología de la Educación (Del Caño y Marugán, 2004; Del Caño, Marugán, Román, Torres y Galán, 2005; Marugán, Del Caño, Román y Torres, 2008; Marugán, Del Caño, Palazuelo y Carbonero, 2010; Palazuelo, Marugán, Del Caño, Frutos y Quintero, 2010). La finalidad última del citado Proyecto está en intervenir en el desarrollo y potenciación de las habilidades creativas de los alumnos, ya que la investigación ha demostrado que la creatividad puede mejorar a través de la estimulación (Garaigordobil, 2005; López, 2000; Llanos, 2002; Prieto, 2002;), al mismo tiempo que mejorar habilidades cognitivas (Chávez, Zacatelco y Acle, 2009). Concretamente se está trabajando con alumnado de altas capacidades a través del proyecto SICO (Palazuelo, Elices y Del Caño, 2007) y en la definición de actuaciones del maestro creativo desde el punto de vista de la motivación, metodología y evaluación (Martín, Marugán y Navarro, 2010).

\section{Objetivos e hipótesis}

Para el trabajo que aquí se presenta, y como revisión actualizada de todo lo dicho, se propusieron los siguientes objetivos: (1) Analizar las diferencias en los resultados, globales y por factores, obtenidos en una prueba de creatividad gráfica por sujetos de alta capacidad intelectual y sujetos que no son de alta capacidad; (2) determinar si todos los sujetos de alta capacidad intelectual obtienen resultados elevados en una prueba de creatividad gráfica. En consonancia con dichos objetivos, se intentaron contrastar las siguientes hipótesis: (a) Existirán 
diferencias significativas en los resultados medios de la prueba de creatividad, globales y por factores, entre los sujetos de alta capacidad intelectual y el resto de participantes, sin alta capacidad. (b) Existirán diferencias significativas en los resultados de la prueba de creatividad, globales y por factores, entre los sujetos muy creativos (sin alta capacidad intelectual) y los sujetos de alta capacidad intelectual.

\section{Método}

\section{Participantes}

El estudio se ha realizado con una muestra de 477 estudiantes de $4^{\circ}, 5^{\circ}$ y $6^{\circ}$ de Educación Primaria (E.P.) y de $1^{\circ}$ y $2^{\circ}$ de Educación Secundaria Obligatoria (E.S.O.) de cuatro centros educativos, dos públicos y dos concertados, con un nivel sociocultural medio. Estos centros habían colaborado en fases anteriores del proyecto de investigación. Se consideró alumno de alta capacidad intelectual ("CI alto") a los sujetos con un CI igual o superior al percentil 95 en una prueba de inteligencia no verbal: el Test de Factor g de Cattell. En este estudio se contó con 54 sujetos identificados como de alta capacidad intelectual.

\section{Tabla 1: Características de la muestra}

\begin{tabular}{llllll}
\hline & & & & & \\
Nivel & $\mathrm{n}$ & $\%$ & Sexo & $\mathrm{n}$ & $\%$ \\
$4^{\circ} \mathrm{EP}$ & 122 & 25,6 & Hombre & 253 & 53 \\
$5^{\circ} \mathrm{EP}$ & 115 & 24,1 & Mujer & 224 & 47 \\
$6^{\circ} \mathrm{EP}$ & 131 & 27,5 & & & \\
$1^{\circ} \mathrm{ESO}$ & 59 & 12,4 & $\mathrm{Cl}$ & $\mathrm{n}$ & $\%$ \\
$2^{\circ}$ ESO & 50 & 10,5 & Alto & 54 & 11,3 \\
N Total & 477 & 100 & No alto & 423 & 88,7 \\
& & & & & \\
\hline
\end{tabular}

\section{Diseño y variables}

Las variables presentes en los análisis de esta investigación son:

1. Capacidad intelectual operativizada en CI alto y CI no alto. A partir de la variable CI, se ha determinado esta variable categórica. Los sujetos de alta capacidad intelectual o “CI alto", son los que tienen un CI por encima o en el percentil 95. Los demás partici- 
pantes, no considerados de alta capacidad intelectual o "CI no alto", tienen un CI por debajo del percentil 95.

2. Creatividad operativizada en una variable cuantitativa procedente de la puntuación global obtenida en la prueba de creatividad TAEC. Se ha categorizado en "Creatividad alta" que agrupa a todos los sujetos con una puntuación global en el TAEC situada por encima o en el percentil 75, y "Creatividad no alta" con puntuaciones por debajo de dicho percentil.

\section{Instrumentos}

Test de Factor $g$ de inteligencia (Escala 2) de R.B. Cattell (1990). Es una prueba de medida de la inteligencia general no verbal de aplicación colectiva, adaptada y tipificada para población española. Consta de tres escalas. La Escala 2, forma A, es la que se empleó en la investigación. Las cuatro pruebas que forman las escalas: Series, Clasificación, Matrices y Condiciones, están elaboradas con elementos perceptivos distintos, con la intención de eliminar en el mayor grado posible las influencias culturales. Al mismo tiempo, al emplear cuatro tareas se pretende evitar que las diferencias de aptitud perceptiva influyan en los resultados.

Test de Abreacción para Evaluar la Creatividad (TAEC) Forma B. (De la Torre, 1991). Se recomienda la utilización de la forma B desde la Educación Primaria en adelante, ya que las figuras a completar son algo más complejas (con mayor número de aperturas). Es una prueba de respuesta gráfica que permite valorar cuantitativamente la creatividad. Se puede aplicar desde los tres años de forma colectiva o individual. La tarea consiste en terminar 12 figuras inacabadas, variadas y poco convencionales. Proporciona una puntuación global y puntuaciones para nueve factores de creatividad. Los factores son: resistencia al cierre de aberturas, originalidad, elaboración de las figuras, fantasía, alcance imaginativo, expansión de las figuras, conectividad de unas figuras con otras, riqueza expresiva o vitalidad de las composiciones y fluidez gráfica. El test ofrece baremación en centiles por niveles educativos.

Se ha elegido esta prueba para valorar creatividad por varios motivos: (a) porque la actividad se puede presentar de una forma lúdica, sin que los sujetos perciban que es un test. Además, carece de límite de tiempo. Estos dos factores, valoración sin presión de evaluación ni de tiempo, fueron considerados por Wallach y Kogan (1965) dos requisitos necesarios para que el sujeto sea realmente creativo en una prueba, (b) La evaluación de nueve factores de 
creatividad, incluyendo factores novedosos más allá de los clásicos de fluidez, flexibilidad, originalidad y elaboración, permite elaborar perfiles de creatividad amplios, lo que concuerda con los objetivos de este trabajo, y (c) La baremación con población española, para los nueves factores de creatividad y para la puntuación global de la prueba, se presta a realizar estudios comparativos entre grupos, tal y como se pretendía en este trabajo de investigación.

Tabla 2. Resumen de los factores de creatividad valorados en la prueba TAEC

\begin{tabular}{|l|l|l|}
\hline $\begin{array}{l}\text { FACTORES DE } \\
\text { CREATIVIDAD }\end{array}$ & QUÉ VALORA & QÚE SE PUNTÚA \\
\hline Resistencia al cierre & $\begin{array}{l}\text { Capacidad para retrasar el cierre de } \\
\text { aperturas en la figura }\end{array}$ & $\begin{array}{l}\text { Aperturas abiertas o cerradas indi- } \\
\text { rectamente }\end{array}$ \\
\hline Originalidad & $\begin{array}{l}\text { Rareza estadística del contenido de la } \\
\text { imagen elaborada }\end{array}$ & $\begin{array}{l}\text { Infrecuencia de la imagen represen- } \\
\text { tada. }\end{array}$ \\
\hline Elaboración & $\begin{array}{l}\text { Enriquecimiento de la imagen con } \\
\text { detalles secundarios y adicionales }\end{array}$ & $\begin{array}{l}\text { Aparición de detalles adicionales, no } \\
\text { necesarios para interpretar la imagen }\end{array}$ \\
\hline Fantasía & $\begin{array}{l}\text { Contenido de la imagen inusual, ale- } \\
\text { jado del entorno familiar }\end{array}$ & $\begin{array}{l}\text { Grado en que la imagen se aleja del } \\
\text { entorno cotidiano }\end{array}$ \\
\hline Conectividad & $\begin{array}{l}\text { Integración de dos o más figuras en } \\
\text { un todo }\end{array}$ & Número de figuras conectadas \\
\hline Alcance imaginativo & $\begin{array}{l}\text { Lugar que ocupa en la imagen la figu- } \\
\text { ra dada: cuerpo principal o elemento } \\
\text { secundario }\end{array}$ & $\begin{array}{l}\text { Grado en que la figura es un ele- } \\
\text { mento secundario de la imagen }\end{array}$ \\
\hline Expansión figurativa & $\begin{array}{l}\text { Espacio ocupado por la imagen elabo- } \\
\text { rada }\end{array}$ & $\begin{array}{l}\text { Grado en que la imagen trasciende } \\
\text { el espacio de cada figura }\end{array}$ \\
\hline Riqueza expresiva & $\begin{array}{l}\text { Colorido, perspectiva y representación } \\
\text { de seres vivos o en movimiento }\end{array}$ & $\begin{array}{l}\text { Presencia de color, volumen o de } \\
\text { seres vivos o en movimiento }\end{array}$ \\
\hline $\begin{array}{l}\text { Coeficiente de fluidez } \\
\text { gráfica }\end{array}$ & $\begin{array}{l}\text { Capacidad de dar respuestas valiosas } \\
\text { en el menor tiempo }\end{array}$ & $\begin{array}{l}\text { Cociente entre las puntuaciones de } \\
\text { los factores anteriores y el tiempo de } \\
\text { realización }\end{array}$ \\
\hline
\end{tabular}

Fuente: elaboración propia

\section{Procedimiento}

Una vez fijados los objetivos y seleccionados los instrumentos, se solicitó la colaboración de los centros educativos en los que se habían detectado sujetos de alta capacidad intelectual. Un psicopedagogo del equipo de investigación se desplazó a los centros para aplicar el test TAEC.

\section{Análisis de datos}

Esta investigación responde a un diseño no experimental descriptivo y analítico, que puede ofrecer una revisión preliminar de hipótesis, de modo que las que sobrevivan a este 
proceso pueden verificarse más tarde de forma experimental (Campbell y Stanley, 2001). El diseño no experimental desarrollado ha combinado un enfoque descriptivo con un enfoque analítico. Se ha recogido información respecto a la variable creatividad, y a nueve indicadores de la misma, con el objetivo de describir dichas variables en una amplia muestra de sujetos, incluido un subgrupo de alta capacidad intelectual.

En todos los contrastes de hipótesis realizados se han empleado tests no paramétricos. Se descartó el uso de tests paramétricos tras comprobar que no se cumplían los supuestos necesarios para aplicarlos: normalidad de la distribución (prueba de Kolmogorv - Smirnov) y homogeneidad de las varianzas entre los grupos comparados (estadístico de Lévene). Los tests elegidos fueron los siguientes: (1) Análisis de correlación variables cuantitivas: Coeficiente de correlación de Spearman. Asociación de variables nominales: prueba Chi - cuadrado. (2) Comparación de dos grupos: Test de la U de Mann - Whitney. (3) Comparación de más de dos grupos: (a) Con varianzas homogéneas: Test de Kruskal - Wallis y analisis post - hoc de Dunn - Sidak. Este test post - hoc aplica un procedimiento de ajuste del error tipo I en comparaciones múltiples que le confiere mayor potencia que el análisis post - hoc de Bonferroni. (b) Varianzas no homogéneas: se transformaron los datos en rangos, aplicando un ANOVA sobre los mismos y un test post - hoc de Scheffé, uno de los análisis post - hoc más conservadores. El nivel de significación elegido para rechazar la hipótesis nula es de $p<0,05$.

\section{Resultados}

La puntuación media de toda la muestra en el TAEC fue de 58,74 (DT =33,49). El grupo de sujetos de alta capacidad intelectual obtuvo unos resultados medios superiores en la prueba de creatividad TAEC $(M=89,30, D T=33,05)$ frente al resto de participantes $(M=$ $54,84, D T=31,51)$. La diferencia existente entre ambos grupos es estadísticamente significativa $(t(65,906)=7,525, p<, 01)$.

Se compararon las puntuaciones medias en todos los factores entre ambos grupos mediante pruebas de $t$ para dos muestras independientes. El TAEC valora nueve factores de creatividad: resistencia al cierre, originalidad, elaboración, fantasía, conectividad, alcance imaginativo, expansión figurativa, riqueza expresiva y coeficiente de fluidez gráfica. En los ocho primeros la puntuación oscila entre 0 y 36 puntos. El coeficiente de fluidez gráfica se calcula dividiendo la suma de las puntuaciones en los demás factores entre el tiempo empleado; en esta investigación la puntuación en ese coeficiente osciló entre 0 y 23 puntos. Las puntuacio- 
nes medias en cada factor para los grupos de CI se recogen en la tabla 3 y gráfico 1 . Se observaron diferencias estadísticamente significativas en todos los factores a favor del grupo de CI alto $(p<, 01)$. Ver Tabla 3.

Tabla 3. Comparación de puntuaciones medias en los factores de creatividad del TAEC (CI alto vs no alto)

\begin{tabular}{lllllll}
\hline & CI & M & DT & t & gl & p \\
\hline Resistencia al cierre & Alto & 15,54 & $(5,10)$ & 3,828 & 82,295 &, 000 \\
\cline { 2 - 7 } & No alto & 12,56 & $(7,16)$ & & & \\
\hline Originalidad & Alto & 13,43 & $(6,20)$ & 4,973 & 475 &, 000 \\
\cline { 2 - 7 } & No alto & 8,41 & $(7,06)$ & & & \\
\hline Elaboración & Alto & 8,83 & $(7,15)$ & 5,760 & 58,085 &, 000 \\
\cline { 2 - 7 } & No alto & 3,10 & $(4,34)$ & & & \\
\hline Fantasía & Alto & 6,67 & $(3,95)$ & 7,153 & 475 &, 000 \\
\cline { 2 - 7 } & No alto & 2,49 & $(4,04)$ & & & \\
\hline Conectividad & Alto & 1,54 & $(2,69)$ & 2,961 & 475 &, 003 \\
\cline { 2 - 7 } & No alto &, 48 & $(2,42)$ & & & \\
\hline Alcance imaginativo & Alto & 11,94 & $(5,77)$ & 7,542 & 475 &, 000 \\
\cline { 2 - 7 } & No alto & 6,31 & $(5,08)$ & & & \\
\hline Expansión figurativa & Alto & 15,39 & $(6,15)$ & 4,200 & 475 &, 000 \\
\cline { 2 - 7 } & No alto & 11,05 & $(7,26)$ & & & \\
\hline Riqueza expresiva & Alto & 9,20 & $(6,09)$ & 4,892 & 474 &, 000 \\
\cline { 2 - 6 } & No alto & 5,55 & $(5,04)$ & & & \\
\hline Coeficiente de fluidez gráfica & Alto & 6,76 & $(2,48)$ & 3,911 & 475 &, 000 \\
\cline { 2 - 6 } & No alto & 4,89 & $(3,39)$ & & & \\
\hline
\end{tabular}

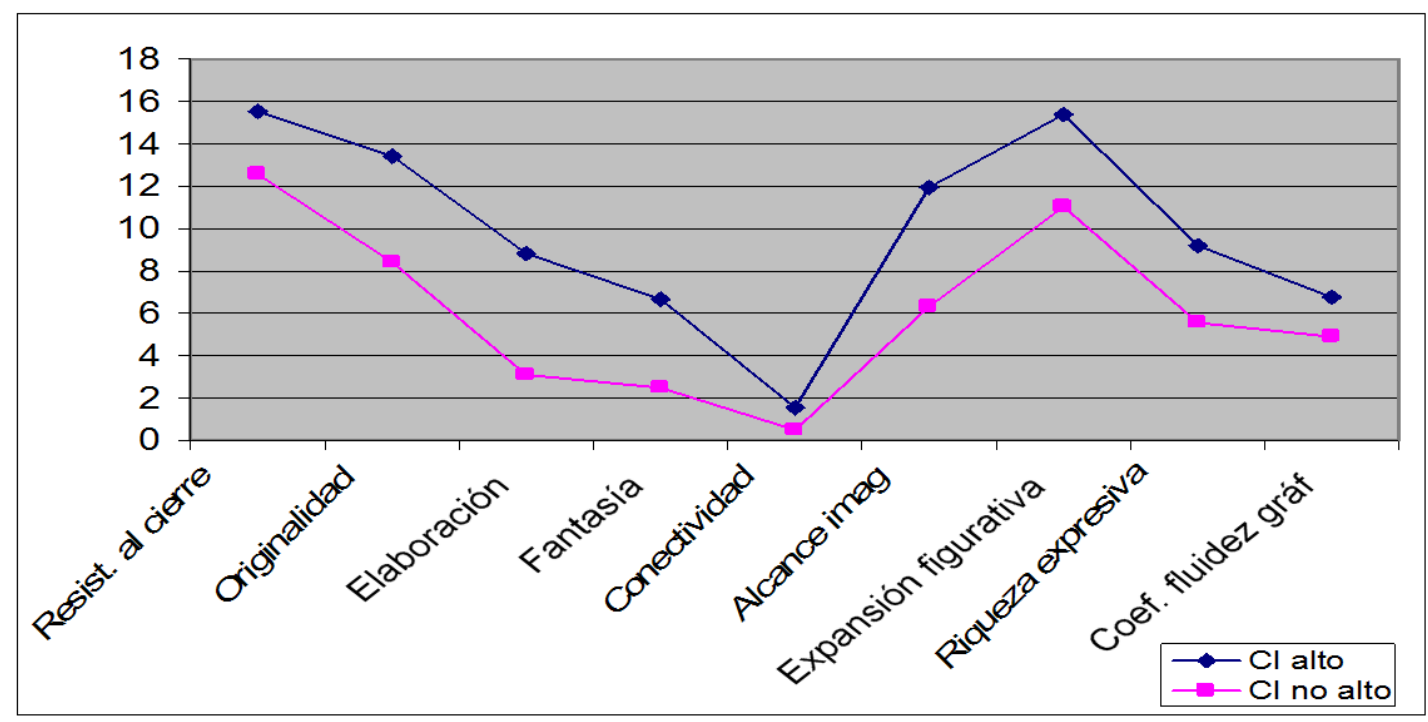

Gráfico 1: Resultados en los factores del TAEC (CI algo vs no alto) 
Para determinar qué sujetos destacan en creatividad se tomó como punto de corte una puntuación superior al percentil 75. Este percentil es aconsejado por diversos autores para valorar creatividad en alumnos superdotados (Castelló, 1999). Un total de 105 participantes superaron dicho percentil, lo que supone un $22 \%$ de la muestra. Se dividió la muestra en cuatro grupos según si los sujetos superaban o no los puntos de corte establecidos en las variables CI (percentil 95) y puntuación en el TAEC (percentil 75). Si superaban el punto de corte se etiquetó el grupo como "alto" y si no lo superaban como "no alto".

Como podemos observar en la tabla 4 , sólo 25 sujetos de alta capacidad intelectual (el 46,3\% del grupo de CI alto) sobrepasaron el punto de corte fijado para la prueba de creatividad. Este subgrupo que destaca en inteligencia y creatividad obtuvo una puntuación media claramente superior a la de los demás subgrupos $(M=113,20, D T=25,580)$. El resto de participantes de alta capacidad intelectual, pero que no superaron el percentil 75 en el TAEC, obtuvieron una media de 68,69 puntos en creatividad $(D T=23,598)$. Destaca también un subgrupo de participantes, un 16,8\% de la muestra, que sin haber superado el punto de corte en CI, sí lo sobrepasa en creatividad, y obtuvo una puntuación media de 98,91 puntos en el TA$\mathrm{EC}(D T=19,752)$.

Tabla 4: Puntuaciones medias en el TAEC por grupos de CI y creatividad

\begin{tabular}{llllll}
\hline Grupos CI x puntuación TAEC & $\mathrm{n}$ & $\mathrm{M}$ & $\mathrm{DT}$ & $\%$ de N & \% grupo de CI \\
\hline CI alto - TAEC alto & 25 & 113,20 & $(25,58)$ & 5,2 & 46,3 \\
CI alto - TAEC no alto & 29 & 68,69 & $(23,59)$ & 6,1 & 53,7 \\
CI no alto - TAEC alto & 80 & 98,91 & $(19,75)$ & 16,8 & 18,9 \\
CI no alto - TAEC no alto & 343 & 44,56 & $(23,97)$ & 71,9 & 81,1 \\
\hline
\end{tabular}

Se analizaron las diferencias entre estos grupos mediante un ANOVA de un factor. Los resultados confirmaron diferencias estadísticamente significativas $(F(3,473)=167.636$, $p<, 01$ ). En las pruebas post hoc (Scheffé) se observó que el grupo de sujetos que destacan en CI y creatividad superan de forma estadísticamente significativa a los otros sujetos de CI alto (tabla 5). En cambio, no existen diferencias estadísticamente significativas con el grupo de sujetos que sin ser de alta capacidad intelectual destaca en creatividad (grupo "CI no alto TAEC alto”). Este grupo sí supera en creatividad, de forma estadísticamente significativa, a los participantes de CI alto que no destacan en el TAEC. 
Tabla 5: Diferencias en puntuaciones medias en el TAEC entre grupos de CI y creatividad

\begin{tabular}{|c|c|c|c|c|c|c|}
\hline \multirow[b]{2}{*}{$\begin{array}{l}\text { Grupos CI y Creativi- } \\
\text { dad }\end{array}$} & & \multirow[b]{2}{*}{$\begin{array}{l}\text { Diferencia } \\
\text { de media }\end{array}$} & \multirow[b]{2}{*}{$\begin{array}{l}\text { Error } \\
\text { típico }\end{array}$} & \multirow[b]{2}{*}{$p$} & \multicolumn{2}{|c|}{$\begin{array}{l}\text { Intervalo de } \\
\text { confianza al 95\% }\end{array}$} \\
\hline & & & & & $\begin{array}{l}\text { Límite } \\
\text { inferior }\end{array}$ & $\begin{array}{l}\text { Límite } \\
\text { superior }\end{array}$ \\
\hline CI alto - TAEC alto & CI alto - TAEC no alto & $44,51(*)$ & 6,383 &, 000 & 26,60 & 62,42 \\
\hline CI alto - TAEC alto & CI no alto - TAEC alto & 14,29 & 5,359 & ,070 &,- 75 & 29,32 \\
\hline CI alto - TAEC alto & CI no alto - TAEC no alto & $68,64(*)$ & 4,845 & ,000 & 55,05 & 82,24 \\
\hline CI alto - TAEC no alto & CI no alto - TAEC alto & $-30,22(*)$ & 5,070 & 000 & $-44,45$ & $-16,00$ \\
\hline CI alto - TAEC no alto & CI no alto - TAEC no alto & $24,13(*)$ & 4,523 & 000 & 11,44 & 36,82 \\
\hline CI no alto - TAEC alto & CI no alto - TAEC no alto & $54,36(*)$ & 2,904 & ,000 & 46,21 & 62,50 \\
\hline
\end{tabular}

\section{Discusión y conclusiones}

Los sujetos de alta capacidad intelectual (CI superior al percentil 95) obtuvieron resultados medios superiores en la puntuación global de la prueba de creatividad gráfico - figurativa TAEC. Asimismo, los participantes de altas capacidades han obtenido mejores resultados en los nueve factores que valora la prueba, tanto en los factores que valoran la elaboración gráfica (resistencia al cierre de aberturas, elaboración de figuras, expansión figurativa y riqueza expresiva), como en los que valoran el contenido de la figura (originalidad, fantasía, alcance imaginativo, conectividad de figuras y coeficiente de fluidez de respuestas). En todos los resultados, global y por factores, las diferencias entre los sujetos de CI alto y el resto de la muestra son estadísticamente significativas $(p<, 01)$.

Si se analiza qué sujetos de la muestra destacan en creatividad (puntuación en prueba superior al percentil 75), no todos los sujetos de CI alto sobrepasan este punto de corte, concretamente, no lo supera un 53,7 \% de los sujetos de alta capacidad intelectual. El subgrupo de participantes de CI alto que sí destaca en creatividad obtiene los mejores resultados medios en la prueba TAEC y supera, de forma estadísticamente significativa, al resto de sujetos de CI alto $(p<.01)$. Estos resultados siguen la línea de estudios clásicos sobre creatividad (Barron y Harrington, 198) y más actuales (López, y Navarro, 2010), en los que las correlaciones entre CI y creatividad eran entre débiles y moderadas, según el campo creativo estudiado. Los resultados de nuestro estudio sugieren que una alta capacidad intelectual permite mejores resultados en la resolución de tareas de creatividad. Sin embargo, no todos los sujetos de alta capacidad intelectual son excepcionalmente creativos. Sternberg (1990) ha afirmado que un CI muy alto no es necesario para ser creativo; incluso, según el mismo autor, una capacidad intelectual muy alta puede impedir el desarrollo de la creatividad, debido a que las fuertes capacidades analíticas de la inteligencia pueden dificultar el proceso creativo. 
La combinación de una prueba de inteligencia no verbal y de creatividad ha facilitado detectar a un grupo de 25 sujetos, potencialmente muy dotados en inteligencia y creatividad, (un 5,2\% de la muestra). Esta combinación de pruebas puede emplearse como sistema de detección inicial o screening. Una evaluación más pormenorizada podría determinar si algunos de los sujetos son superdotados. Al tiempo, se ha detectado un subgrupo de 80 alumnos que, sin ser de alta capacidad intelectual, son altamente creativos y sus resultados en la prueba de creatividad no difieren significativamente del subgrupo de CI y creatividad altos. Estaríamos, pues, ante un grupo de sujetos con una alta capacidad creativa específica, los cuales podrían ser alumnos talentosos. Castelló (1999) define dos tipos de talentos, un talento artístico y un talento creativo, en los que la alta capacidad creativa es un rasgo definitorio.

Este trabajo ha permitido una nueva aproximación al análisis de las relaciones entre inteligencia y creatividad. La combinación de pruebas empleada ha resultado interesante para una detección inicial de los sujetos que destacan en una o ambas facultades, siguiendo los modelos actuales de superdotación y talento. La detección de estos alumnos es un paso previo a la intervención educativa.

Este estudio, sin embargo, sólo ha valorado una vertiente de la creatividad (gráfico figurativa). Se propone un estudio más pormenorizado que incluyera estilos cognitivos, motivación, dominio del campo (en este caso, la composición gráfica) o conocimientos previos. También se debería tener en cuenta otros instrumentos de evaluación de creatividad que incluyen el ámbito verbal, así como otros procedimientos de valoración que completen la evaluación estandarizada (observación, heteroevaluación). Desde el punto de vista de la inteligencia habría que contemplar la perspectiva de las inteligencias múltiples como factores predisponentes de las determinadas habilidades creativas. Y, por supuesto, no habría que olvidar la influencia de las variables contextuales y culturales.

\section{Agradecimientos}

Este trabajo ha sido realizado en el Grupo de Investigación de Excelencia GR179 y ha contado con la financiación de la Junta de Castilla y León. 


\section{Referencias}

Ademola, R., Akintunde, S.O. y Yakasi, M.I. (2010). Inteligencia emocional, creatividad y logro académico en los estudiantes de empresariales. Electronic Journal of Research in Educational Psychology, 8 (2), 763-786.

Alonso, C. (2000). Qué es la creatividad. Madrid (España): Biblioteca Nueva.

Chávez, B., Zacatelco, F. y Acle, G. (2009). Programa de enriquecimiento de la creatividad para alumnas sobresalientes de zonas marginadas. Electronic Journal of Research in Educational Psychology, 7(2), 849-876.

Barron, F. y Harrington, D. M. (1981). Creatividad, inteligencia y personalidad. Annual Review of Psychology, 32, 439-476.

Bermejo, M. R., Castejón, J. L. y Sternberg, R. J. (1996). Insight in children with high intelligence level. FAISCA, 4, 85-94.

Campbell, D. y Stanley, J. (2001). Diseños experimentales y cuasiexperimentales en la investigación social. Buenos Aires: Amorrortu.

Castelló, A. (1999). Alumnat excepcionalment dotat intellectualment. Barcelona (España): Generalitat de Catalunya.

Cattel, R.B. y Cattel, A.K.S (1990). Test de Factor “g”. Escala 2. Madrid (España): TEA Ediciones S.A.

Davidson, J. E. \& Sternberg, R. J. (1986). The role of insight in giftedness. Gifted Child Quarterly, 28, 58-64.

Del Caño, M. y Marugán, M. (2004). Identificación de niños y niñas superdotados en la Comunidad Autónoma de Castilla y León. Fase I. En M.I. Ruiz, F. Fajardo y A.V. Vicente (Eds.). Aportaciones Psicológicas y desarrollo difícil (pp. 31-41). Badajoz (España): Editex.

Del Caño, M., Marugán, M., Román, J. M., Torres, H. y Galán, M. (2005). Estrategias de aprendizaje y alumnos con altas capacidades. En M.I. Ruiz, F. Fajardo \& A.V. Vicente (Eds.). Necesidades educativas específicas (pp. 65-72). Badajoz (España): Editex.

Ferrando, M., Prieto, M.D., Ferrándiz, C. y Sánchez, C. (2005). Inteligencia y creatividad. Electronic Journal of Research in Educational Psychology, 7 (3), 21-50

Garaigordobil, M. (2005). Evaluación del cambio de conducta y rasgos de personalidad creadora: efectos de una experiencia con niños de 10 - 11 años. Infancia y Aprendizaje, 28 (1), 51-61.

Guilford, J. P. (1967). The nature of human intelligence. Traducción española: La naturaleza de la inteligencia humana. Barcelona (España): Paidós, 1986.

Llanos, M. D. (2002). Intervención psicoeducativa en el ámbito de la creatividad. Aplicación y evaluación de un programa para el desarrollo del pensamiento creativo en la educación primaria. Madrid (España): UNED.

López, C. (2000). Creatividad en niños superdotados. Tesis doctoral. Universidad Complutense de Madrid (España).

López, O. (2001). Evaluación y desarrollo de la creatividad. Tesis doctoral. Universidad de Murcia (España).

López, O. y Navarro, J. (2010). Creatividad e inteligencia: un estudio en Educación Primaria. Revista de Investigación Educativa, 28 (2), 283-296.

Martínez, J. M. y Rimm, S. (1985). Cuestionarios de creatividad. Madrid (España): San Pío $\mathrm{X}$. 
Marugán, M., Del Caño, M., Román, J. M. y Torres, H. (2008). Estudio comparativo entre la evaluación psicométrica y la identificación del profesor para variables psicoeducativas relevantes en Primaria y Secundaria. Revista de Psicología General y Aplicada, 60 (4), 377-396.

Marugán, M., Del Caño, M., Palazuelo, M. y Carbonero, M.A. (2010). Percepción creativa de la ciudad en niños y jóvenes. Estudio empírico y propuesta de actuación. Aula Abierta, $38,37-46$

Martín, C., Marugán, M. y Navarro, J. I. (2010). Creatividad y educación. En Navarro, J. I. y Martín, C. (Coords.), Psicología de la Educación para docentes (pp. 89-108). Madrid (España): Pirámide.

Matud, P. (1999). Creatividad, inteligencia y personalidad en estudiantes de enseñanzas medias y en universitarios: un estudio psicométrico. En A. Sipán (Coord.), Respuestas educativas para alumnos superdotados y talentosos (pp. 345-352). Zaragoza (España): Mira Editores.

Monreal, C. (2000). Qué es la creatividad. Madrid (España): Biblioteca Nueva.

Palazuelo, Ma.M., Elices, J.A. y Del Caño, M. (2007) Alumnado con superdotación: respuesta educativa. Valladolid (España): Junta de Castilla y León.

Palazuelo, M., Marugán, M., Del Caño, M., De Frutos, C. y Quintero, M. (2010). La expresión emocional en alumnos de altas capacidades. Faisca: Revista de Altas Capacidades, 15 (17), 50-66.

Preckel, F., Holling, H. y Wiese, M. (2006). Relationship of intelligence and creativity in gifted and non - gifted students: An investigation of threshold theory. Personality and Individual Differences, 40, 159-170.

Prieto, M. D., López, O., Bermejo, M. R., Renzulli, J. S. y Castejón, J. L. (2002). Evaluación de un programa de desarrollo de la creatividad. Psicothema, 14(2), 410-414.

Renzulli, J. S. (1999). What is this thing called giftedness, and how do we developed it? A twenty - five year perspective. Journal for the education of the gifted, 23(1). 3-54

Sligh, A., Conners, F., Roskos, B. (2005). Relation of creativity to fluid and crystallized intelligence. The Journal of Creative Behaviour, 39(2), 123-136.

Sternberg, R. J. (1985). Beyond IQ: a triarchic theory of human intelligence. Nueva York: Cambridge University Press.

Sternberg, R. J. (1990). Más allá del cociente intelectual. Bilbao (España): DDB.

Sternberg, R. J. y Lubart, T. I. (1997). La creatividad en una cultura conformista. Un desafio a las masas. Barcelona (España): Paidós.

Sternberg, R. J. y O’hara, L. A. (1999). Creativity and Intelligence. En R. J. Sternberg (Ed.) Handbook of creativity (pp. 251-272). Nueva York: Cambridge University Press.

Torrance, E. P. (1975). Creativity research in education: still alive. En I. A. Taylor y W. Getzels (Eds.), Perspectives in creativity (pp. 165-181). New Brunswick (EE.UU.): Transaction Books

Torre, S. de la (1991). Evaluación de la creatividad: un instrumento de apoyo a la reforma. Madrid (España): Escuela Española.

Vallach, M.A. y Kogan, N. (1965). Modes of thinking in Young children. Nueva York: Holt, Rinehart \& Winston.

Ward, T. B., Saunders, K. y Dodds, R. (1999). Creative cognition in gifted adolescents. Roeper Review, 21(4), 260-266. 